\title{
ALTENGLISCHES AUS LEIDENER HANDSCHRIFTEN.
}

\author{
Aus Voss. Lat. $4^{0}$ No. 106. 9. Jhd.
}

Demselben schreiber dieses Codex der Rätsel von Symphosius-Aldhelm, der uns am ende desselben das sog. Leidener Rätsel aufbewahrt hat, verdanken wir auch eine liste ae. nymphennamen, zu deren niederschrift er den leeren raum der zweiten hälfte von folio 10 recto benutzt hat. Sie sind zwar schon 1845 von Bethmann im fünften bande der ZfdA., seite 199, der öffentlichkeit übergeben worden, verdienen aber trotzdem einen neuabdruck in der Anglia, weil sie da, wo sie veröffentlicht waren, augenscheinlich der beachtung der Anglisten entgangen sind: weder hat Sweet sie in seine OET. aufgenommen, noch auch hat das NED. unter clven 'female elf' sie berücksichtigt, obwohl sie zwei jahrhunderte ältere belege für das wort enthalten und besser geeignet waren, es zu illustrieren, als die liste des spätae. supplements zu Filfrics Focabular (WW. $189^{4-y}$ ), der allein die ae. belege entnommen sind. Ich drucke genau nach der hs. ${ }^{1}$ und stelle die liste des Vocubulars daneben.

fol. $10 \times 2$

1s Nimphae ${ }^{1}$ ) . aetfunn eadem $\left.{ }^{2}\right) \&^{3}$ ) muse

1: Oreades ${ }^{1}$ ) duun . aetfinn . . . Oreades . muntalfen, WW. 189'. Castalides . dınelfen, WW. $189^{9}$.

20 Driades ${ }^{1}$ ) uudu . aetfinue . . . Dryades . wuduelfen, WW.1895.

21 A'madriades $^{1}$ ) ua\&er ${ }^{4}$ ) . aetfuñ $\tilde{n}^{5}$ ) Hamadryades . wylde elfen, WW. 189?.

2? Maides 1) feld . ael finne ${ }^{6}$ ) . . . Moides . feldelfen, WW.1896.

2.3 Taides ${ }^{1}$ ). sâe ${ }^{i}$ ) aetfinne . . . Naiades . sceelfen., WW. $189^{\text {s. }}$

1) Das $a$ ist das sog. offene. 2) So! für ecclem. $\quad$ ") Bethmann: et.

4) Bethmann: uaeter. 5) Bethmann löst auf, ohne bemerkung, aelfinne

๑) Bethmann: aelfinne. ') Bethmann läfst den akzent weg. 
Man beachte, dafs in dieser liste sich die "älteste und eigentliche endung des nom. pl." (Sievers, Ags. Gr. ${ }^{3}, \S 269$, anm. 2), $i$ neben der jüngeren, $e$, findet. Beachte auch die abkürzung von $e$ durch die wellenlinie über $n$ in aelfinn. Beachte ferner $u=u u=w$.

Wenn in der Leidener aufzählung die hamadryaden als 'wasserelben' bezeichnet werden, während das Vocabular sie richtig als 'waldelben' angibt, so beruht das jedenfalls darauf, dals in irgend einem stadium der vorlage ein ausfall stattgefunden hat. Mich dünkt, es stand einst so:

Dryadas . uudu . aelfinne

Hamadryades idem .

Castalides 1) dunn . $¥$ uuaeter aelfinne.

Bemerkenswert ist der lateinische name der feldelben, maides. Das ist olne zweifel eine keltisch-lateinische bildung von kelt.-ir. mag 'campus', die der von ogastrum im Epinal analog ist, das ae. aeggemong erklärt wird und somit auf ir. og 'ovum' weist. Ähnlich steht es ebenda mit prifeta, das ae. thriuuintri steor erklärt wird und somit auf $\bar{p}_{\text {rifeta, lat. }}$ *triueta $=$ gr. $\tau \rho \iota \varepsilon \tau \dot{\eta} \varsigma$ zurückgehen muls. Das ist zugleich ein neuer beweis für die richtigkeit der von mir seit jahren vertretenen ansicht, dafs schon in der vorlage des Epinal die thorn-rune verwandt wurde, wie sie denn im Epinal selbst keineswegs so selten ist, als man es hat hinstellen wollen, und alle darauf gebauten theorien linfällig sind.

1) Im Læceboc ed. Lconhardi, p. $106^{34}$, scheinen sie ganz allgemein für krankheit verursachende elben $z u$ stehen: Deus omnipotens ......... expelle a famulo tuo $\mathrm{N}$. Omnem impetū (so!) castalidum de capite, de capillis ........ de conpaginibus . omnium membrorum . intus et foris.

Basel, Mai 1912.

Otto B. Schlutter. 